\title{
Altered Extended Locus Coeruleus and Ventral Tegmental Area Networks in Boys with Autism Spectrum Disorders: A Resting-State Functional Connectivity Study
}

\author{
Yiting Huang ${ }^{1,2}$ \\ Siyi $\mathrm{Yu}^{2}$ \\ Georgia Wilson ${ }^{2}$ \\ Joel Park ${ }^{2}$ \\ Ming Cheng ${ }^{2}$ \\ Xuejun Kong ${ }^{3}$ \\ Tao Lu' \\ Jian Kong ${ }^{2}$
}

'School of Life Sciences, Beijing University of Chinese Medicine, Beijing,

People's Republic of China; ${ }^{2}$ Department of Psychiatry, Massachusetts General Hospital, Harvard Medical School, Boston, MA, USA; ${ }^{3}$ Martino Imaging Center, Massachusetts General Hospital, Harvard Medical School, Boston, MA, USA

\begin{abstract}
Introduction: Previous studies have suggested that cerebral projections of the norepinephrine (NE) and dopamine (DA) systems have important etiology and treatment implications for autism spectrum disorder (ASD).

Methods: We used functional magnetic resonance imaging to evaluate spontaneous resting state functional connectivity in boys aged 7-15 years with ASD $(n=86)$ and age-, intelligence quotient-matched typically developing boys (TD, $n=118$ ). Specifically, we investigated functional connectivity of the locus coeruleus (LC) and ventral tegmental area (VTA), the main source projection of neurotransmitters NE and DA, respectively.

Results: 1) Both the LC and VTA showed reduced connectivity with the postcentral gyrus (PoCG) in boys with ASD, reflecting the potential roles of NE and DA in modulating the function of the somatosensory cortex in boys with ASD. 2) The VTA had increased connectivity with bilateral thalamus in ASD; this alteration was correlated with repetitive and restrictive features. 3) Altered functional connectivity of both the LC and VTA with brain regions such as the angular gyrus (AG), middle temporal gyrus visual area (MT/V5), and occipital face area (OFA) in ASD group.

Discussion: Our findings implicate the role of LC-NE and VTA-DA systems from the perspective of functional neuroimaging and may shed light on pharmacological studies targeting NE and DA for the treatment of autism in the future.
\end{abstract}

Keywords: autism spectrum disorder, locus coeruleus, ventral tegmental area, norepinephrine, dopamine, functional connectivity

\section{Introduction}

Autism spectrum disorder (ASD) encompasses a set of neurodevelopmental disorders characterized by repetitive and restrictive behavioral phenotypes and deficits in social and cognitive functions. ${ }^{1}$ Recently, there has been a surge in the recognition and diagnosis of ASD, and the prevalence rates are estimated at $1 \%$ to $2 \%{ }^{2}$ Currently, pharmacological treatment for ASD is still challenging.

The noradrenergic system, with widely distributed axons throughout the central nervous system (CNS), is critical for maintaining the waking and vigilant state among other functions. Within the waking state, this system also enhances the collection and processing of salient sensory information via actions on sensory, memory, attentional, and motor processes. ${ }^{3}$ The locus coeruleus is the principal site
School of Life Sciences, Beijing University of Chinese Medicine, No. II East Road North 3rd Ring Road, Beijing, 100029, People's Republic of China

Email taolu@bucm.edu.cn

Jian Kong

Department of Psychiatry, Massachusetts General Hospital, Harvard Medical

School, I20 2nd Ave, Room I0IC,

Charlestown, MA, 02129, USA

Email jkong2@mgh.harvard.edu 
for brain synthesis of NE. ${ }^{4}$ Previous literature has regarded the locus coeruleus-norepinephrine (LC-NE) system as a pacemaker of attentional function. ${ }^{5,6}$ Bast et al recently proposed that aberrant attentional function through atypical LC-NE activity in ASD may be associated with reduced social attention and interactions. ${ }^{7}$

The dopaminergic system accounts for less than $1 \%$ of axons throughout the CNS, yet the system has a profound function in the brain. DA was proven to be related to motor control, reward, motivation, and cognition. ${ }^{8-10}$ The dopaminergic system in the ventral tegmental area (VTA) is a key region involved in reward processing. ${ }^{11}$ Abrams and colleagues reported underconnectivity between the voice-selective area and several distributed nodes of the reward pathway in children with $\mathrm{ASD}{ }^{12}$ The authors suggested that ASD subjects may experience a less pleasurable response to human voice processing, thus impacting language and social skills. Supekar and his team found that deficits in the mesolimbic reward pathways may be linked with the impaired social skills of children with autism. ${ }^{13}$

Studies suggest that these two structures can be both independent and complementary. ${ }^{14,15}$ Sometimes DA and NE signaling may be involved in functionally opposing processes. For instance, an aversive stimulus may activate noradrenergic signaling but inhibit dopaminergic signaling, whereas a rewarding stimulus may inhibit norepinephrine while stimulating dopamine release. ${ }^{16}$ On the other hand, the NE and DA systems also interact functionally. NE transmission is essential for DA release, ${ }^{17-19}$ and both the LC-NE and VTA-DA systems extensively innervate the prefrontal cortex and are influential in regulating prefrontal cortex (PFC)-dependent functions ${ }^{20}$ as well as the attention-related default mode network (DMN). ${ }^{21-23}$

Over the past few decades, numerous studies have attempted to identify the specific neurometabolic disorders in children with $\mathrm{ASD}^{24}$ Evidence of the relationship between norepinephrine (NE), dopamine (DA), and ASD was gathered from studies reporting decreased dopamine B hydroxylase activity and increased serum NE levels in children with ASD and their parents. ${ }^{25}$ The important role of brain NE and DA in autism spectrum disorder has recently been proposed. ${ }^{26,27}$ It is worth noting that the most conventional and atypical antipsychotics currently approved for ASD target the DA system, and blocking the NE system has been shown to benefit social and language functions in single-dose psychopharmacological trials with propranolol in individuals with $\mathrm{ASD}^{28,29}$
Effects of beta-blockers in reducing autism-related emotional and behavioral dysregulation were also reported in a series of case studies. ${ }^{30,31}$ Additionally, there are studies exploring the effects of propranolol on functional connectivity. ${ }^{32,33}$

To date, few studies have aimed to elucidate the interactive function of the LC and VTA in ASD populations from the perspective of brain neuroimaging. The current study therefore characterized resting-state functional networks of LC and VTA in boys with ASD compared to a typically developing (TD) group using a seed-based approach. To confirm potential relationships between functional networks and behavior, we also explored the association between the available behavioral outcome measures and imaging findings. We hypothesized that ASD is associated with altered functional connectivity of the LC and VTA and that this alteration may be associated with behavioral deficits in social interactions of children with ASD.

\section{Materials and Methods Subjects}

Datasets were extracted from the Autism Brain Imaging Data Exchange II (ABIDE II, http://fcon_1000.projects. nitrc.org/indi/abide/abide II.html). To study the connectivity-behavior correlation, we chose sites that shared behavioral measurements to the greatest extent. All subjects used in this manuscript were obtained from five institutions [Georgetown University (GU), Kennedy Krieger Institute (KKI), New York University (NYU), San Diego State University (SDSU), and Trinity Center for Health Sciences (TCD)]. All procedures from these five selected sites were approved by their local Institutional Review Boards.

Inclusion criteria were: i) boys aged 7 to 15 years old to minimize potential developmental effects; ${ }^{34,35}$ ii) fullscale IQ (F-IQ) score $>80$; iii) right-handedness; iv) scanned in a $3 \mathrm{~T}$ MRI scanner to increase between-site reliability; v) diagnosis of ASD based on DSM-IV-TR and assessed with the Autism Diagnostic Observation Schedule (ADOS), the Autism Diagnostic InterviewRevised (ADI-R), or both. All individuals with ASD completed the Social Response Scale (SRS), a 65-item rating scale that quantifies severity of ASD. ${ }^{36}$

\section{fMRI Imaging Acquisition}

Each of the participants received resting-state fMRI and anatomical scans acquired on 3T scanners: Siemens Trio 
3T scanner, GU; Philips 3T scanners, KKI; Siemens Allegra, NYU; GE 3T MR750 scanner, SDSU; Philips Intera Achieva 3T MR system, TCD. The detailed scan procedures and parameters are described on the ABIDE website (http://fcon 1000.projects.nitrc.org/indi/abide/).

\section{fMRI Data Analysis}

In this study, we want to investigate the role of norepinephrine (NE) and dopamine (DA) systems in ASD, we thus have decided to choose a hypothesis-driven method (a seedbased approach using LC and VTA as seed). Functional MRI data was preprocessed and analyzed using the CONN toolbox v.18.a, ${ }^{22}$ a Matlab/SPM-based cross-platform software (https://www.nitrc.org/projects/conn). fMRI data were preprocessed using the default pipeline in CONN. The preprocessing steps included slice time correction, realignment, segmentation of structural data, spatial and functional normalization into standard stereotactic Montreal Neurosciences Institute (MNI) space, spatial smoothing (Gaussian kernel of $6 \mathrm{~mm}$ FWHM) and resliced to $2 \times 2 \times$ $2 \mathrm{~mm}$ voxels. Subjects whose head motion exceeded the group mean (mean relative root mean square [RMS] displacement) $+2 \times$ group standard deviation (SD) (mean relative RMS displacement) were excluded. ${ }^{37,38}$

To further eliminate correlations caused by head motion and artifacts, we identified outlier time points in the motion parameters and global signal intensity using ART implemented in CONN toolbox (https://www.nitrc.org/projects/ artifact_detect/). For each subject, we treated images as outliers if composite movement from a preceding image exceeded $0.5 \mathrm{~mm}$, or if the global mean intensity was $>3$ SDs from the mean image intensity for the entire resting scan. The outliers were used for subsequent scrubbing regression. The structural images were segmented and used to create gray matter, white matter (WM), and cerebral spinal fluid (CSF) masks of each subject. Then, linear regression using WM \& CSF signals, linear trend, subject motion ( 6 rotation/translation motion parameters and 6 firstorder temporal derivatives), and outliers (scrubbing) was conducted to remove confounding factors. After that, the residual BOLD time series was band-pass filtered with a frequency window of $0.008-0.9 \mathrm{~Hz}$.

The LC complex was defined based on the previous work of Tona et al. ${ }^{39}$ We extracted the overlay from the four test-retest masks they published (https://www.nitrc.org/ projects/prob lc 3t) and combined the extracted left and right masks into a bilateral LC seed using xjView toolbox (http://www.alivelearn.net/xjview). Based on previous studies, ${ }^{40-43}$ the VTA was defined as a bilateral $4 \mathrm{~mm}$ radius sphere centered at left $(-4,-15,-9)$ and right $(5,-14,-8)$ regions of interest (ROI) in MNI space using WFU-Pick Atlas software (http://fmri.wfubmc.edu/software/pickatlas).

First-level correlation maps were produced by extracting the average Blood-Oxygen-Level-Dependent (BOLD) time courses from each LC complex and VTA seed and by computing Pearson's correlation coefficients between that time course and the time courses of all other voxels in the brain. Correlation coefficients were Fisher transformed into "Z" scores, which increased normality and allowed for improved second-level general linear model analyses.

We first applied one sample $t$-tests to explore the positive and negative functional connectivity within each group using a voxel-wise threshold of $p<0.005$ with $p<0.05$ cluster level False Discovery Rate (FDR)-corrected. Then, a voxel-wise threshold of $p<0.005$ with $p<0.05$ cluster level FDRcorrected was applied for between group comparisons. Age, FIQ, data site, and mean relative RMS were included in the analysis as covariates of non-interest.

We also extracted parameter estimates for all brain regions that showed significant between-group (ASD vs TD) differences and investigated the correlation between extracted cluster parameter estimates and scores for the Social Responsiveness Scale (SRS) and the Autism Diagnostic Interview-Revised (ADI-R) in the ASD group using a partial correlation. We did not include ADOS for the correlation analysis as some sites used original ADOS$\mathrm{G}$ while others used the updated ADOS-2. Age, F-IQ, data site, and mean relative RMS were also included as covariates of non-interest. Bonferroni correction was used to correct for multiple comparisons.

\section{Results}

\section{Demographic and Clinical Characteristics}

Two hundred and sixteen male subjects were included in the study. Based on the head motion criteria, 12 subjects were excluded. In total, 204 male subjects (ASD group: $\mathrm{n}=86$; TD group: $\mathrm{n}=118$ ) were included in the functional connectivity analysis. There were no significant differences between the two groups in terms of age $(p=0.12)$, F-IQ $(p=0.71)$, and mean relative RMS $(p=0.21)$. Interaction effects between site and group were not significant in age $[\mathrm{F}(4,194)=0.599$; $\mathrm{p}=0.664]$, F-IQ $[\mathrm{F}(4,194)=2.395 ; \mathrm{p}=0.052]$ or mean relative RMS $[F(4,194)=0.542 ; p=0.705]$. Detailed demographic and clinical characteristics for all participants included in the study are shown in Table 1. 
Table I Participant Demographics and Clinical Characteristics

\begin{tabular}{|c|c|c|c|}
\hline Characteristic & ASD $(n=86)$ & $T D(n=118)$ & $P$ value \\
\hline Age & $11.0 \pm 1.9$ & $10.6 \pm 1.6$ & 0.12 \\
\hline Full-scale IQ & $112.5 \pm 13.2$ & $113.1 \pm 10.0$ & 0.71 \\
\hline Head motion & $0.15 \pm 0.08$ & $0.13 \pm 0.09$ & 0.21 \\
\hline \multicolumn{4}{|l|}{ ADI-R } \\
\hline Social interaction & $18.7 \pm 5.6$ & - & - \\
\hline Verbal communication & $14.7 \pm 4.6$ & - & - \\
\hline$\nmid$ Non-verbal communication & $7.8 \pm 3.4$ & - & - \\
\hline RRB & $5.6 \pm 2.4$ & - & - \\
\hline \multicolumn{4}{|l|}{ SRS } \\
\hline Total & $76.1 \pm 11.6$ & $43.1 \pm 5.7$ & $<0.001$ \\
\hline Awareness & $69.7 \pm 11.3$ & $44.3 \pm 8.6$ & $<0.001$ \\
\hline Cognition & $71.3 \pm 12.1$ & $42.6 \pm 5.4$ & $<0.001$ \\
\hline Communication & $74.6 \pm 12.5$ & $43.4 \pm 6.0$ & $<0.001$ \\
\hline Motivation & $70.4 \pm 13.0$ & $46.0 \pm 7.1$ & $<0.001$ \\
\hline Mannerism & $76.6 \pm 14.5$ & $44.5 \pm 5.3$ & $<0.001$ \\
\hline
\end{tabular}

Note: ${ }^{\dagger}$ Indicates 15 missing data.

Abbreviations: ADI-R, Autism Diagnostic Interview; SRS, Social Responsiveness Scale; RRB, restrictive and repetitive behavior; mannerism, restricted interests, and repetitive behavior.

\section{Seed-to-Voxel Functional Connectivity} Results

\section{Functional Connectivity with LC Complex as Seed}

In the TD group, we found that the LC had positive restingstate functional connectivity ( $\mathrm{rsFC}$ ) with the bilateral pons, midbrain, cerebellum (anterior/superior posterior lobe), thalamus (lateral nuclei), parahippocampal gyrus (PHG), amygdala, hippocampus, insula, anterior cingulate cortex (ACC), cuneus, precuneus, fusiform gyrus, and lingual gyrus, and negatively correlated with the bilateral middle frontal gyrus, cerebellum, inferior temporal gyrus (ITG), middle temporal gyrus (MTG), left precentral gyrus (PreCG), supramarginal gyrus (SMG), right postcentral gyrus (PoCG), and medial orbital gyrus (see Figure $\mathrm{S} 1$ and Table S1 in supplementary materials). Similar results were observed in the ASD group (see Figure S2 and Table S1 in supplementary materials).

Statistical comparisons between the TD and ASD groups showed that boys with ASD had decreased LC connectivity with the right lingual gyrus and angular gyrus (AG), left postcentral gyrus (PoCG), and cerebellum, and increased LC connectivity with the bilateral posterior middle temporal visual area (MT/V5) compared to the TD group (Table 2; Figure 1).

There is no significant correlation found between LC connectivity and ADI-R and SRS behavior parameters in boys with ASD after Bonferroni correction.

\section{Functional Connectivity with VTA as Seed}

In the TD group, we found that the VTA showed a positive rsFC with the bilateral midbrain, pons, thalamus (lateral nuclei), ACC, medial prefrontal cortex (mPFC), PHG, hippocampus, cuneus, lingual gyrus, operculum, IFG, insula, PoCG, PreCG, SMA, putamen, and amygdala, and showed a negative rsFC with the bilateral cerebellum and precuneus (see Figure S3 and Table S1 in supplementary materials). Similar findings were detected in the ASD group (see Figure $\underline{\mathrm{S} 4}$ and Table $\mathrm{S} 1$ in supplementary materials).

Statistical comparisons between the TD and ASD groups showed that boys with ASD had decreased VTA functional connectivity with the right occipital face area (OFA) and left postcentral/supramarginal gyrus (PoCG/SMG), and increased VTA functional connectivity with the bilateral thalamus compared to TD group (Table 2; Figure 1).

In boys with ASD, rsFC between the VTA and bilateral thalamus was found to positively correlate with the SRS repetitive and restrictive behaviors subscore $(\mathrm{r}=0.3$, $\mathrm{p}=0.004$, Figure 2 ), significantly after Bonferroni correction $(\mathrm{p}=0.05 / 10=0.005)$.

\section{Discussion}

In this study, we explored the resting-state functional connectivity of the LC and VTA, two main sources of $\mathrm{NE}$ and DA, in boys with ASD and TD groups. We found that: 1) both the LC and VTA had a reduced 
Table 2 Comparison of rsFC in the LC and VTA Seeds Between TD and ASD Groups

\begin{tabular}{|c|c|c|c|c|c|c|c|}
\hline \multirow[t]{2}{*}{ Seed } & \multirow[t]{2}{*}{ Condition } & \multirow[t]{2}{*}{ Region (BA) } & \multirow[t]{2}{*}{ Cluster Size } & \multicolumn{3}{|c|}{ MNI Coordinates } & \multirow[t]{2}{*}{ Peak z value } \\
\hline & & & & $\mathbf{x}$ & $\mathbf{Y}$ & $\mathbf{Z}$ & \\
\hline \multirow[t]{6}{*}{ LC } & \multirow[t]{4}{*}{ TD > ASD } & Right lingual gyrus (BAI7) & 226 & 12 & -68 & 0 & 3.89 \\
\hline & & Left PoCG (BAI) & 187 & -30 & -18 & 34 & 4.42 \\
\hline & & Right Angular gyrus (BA39) & $|3|$ & 48 & -58 & 32 & 3.44 \\
\hline & & Left cerebellum (anterior) & 133 & -20 & -26 & -26 & 3.97 \\
\hline & \multirow[t]{2}{*}{$\mathrm{ASD}>\mathrm{TD}$} & Left MT/V5 (BAI9/BA2I) & 236 & -56 & -68 & -6 & 4.48 \\
\hline & & Right MT/V5 (BAI9/BA2I) & 320 & 60 & -58 & 4 & 4.28 \\
\hline \multirow[t]{5}{*}{ VTA } & \multirow[t]{2}{*}{ TD > ASD } & Left PoCG/SMG (BAI/BA40) & 289 & -62 & -20 & 24 & 4.16 \\
\hline & & Right OFA (BAI8) & 465 & 46 & -78 & -8 & 3.77 \\
\hline & \multirow[t]{3}{*}{$\mathrm{ASD}>\mathrm{TD}$} & \multirow[t]{2}{*}{ Bilateral thalamus (pulvinar) } & \multirow[t]{2}{*}{357} & 8 & -30 & 6 & 4.04 \\
\hline & & & & -6 & -28 & 6 & 3.40 \\
\hline & & Right thalamus (ventral anterior) & 150 & 8 & -4 & 6 & 4.7 \\
\hline
\end{tabular}

Abbreviations: rsFC, resting-state functional connectivity; TD, typical developing; ASD, autism spectrum disorders; BA, Brodmann area; LC, locus coeruleus; VTA, ventral tegmental area; MNI, Montreal Neurosciences Institute; PoCG, postcentral gyrus; MT/V5, middle temporal visual area; SMG, supramarginal gyrus; OFA, occipital face area (inferior occipital gyrus).

connectivity with postcentral gyrus in the ASD group, reflecting the potential roles of $\mathrm{NE}$ and DA in modulating the function of the somatosensory cortex in boys with ASD. 2) the VTA had increased connectivity with bilateral thalamus in ASD, this alteration was correlated with repetitive and restrictive features. 3) altered functional connectivity of both the LC and VTA with brain regions such as the angular gyrus (AG), middle temporal gyrus visual area (MT/V5), and occipital face area (OFA) in ASD group.

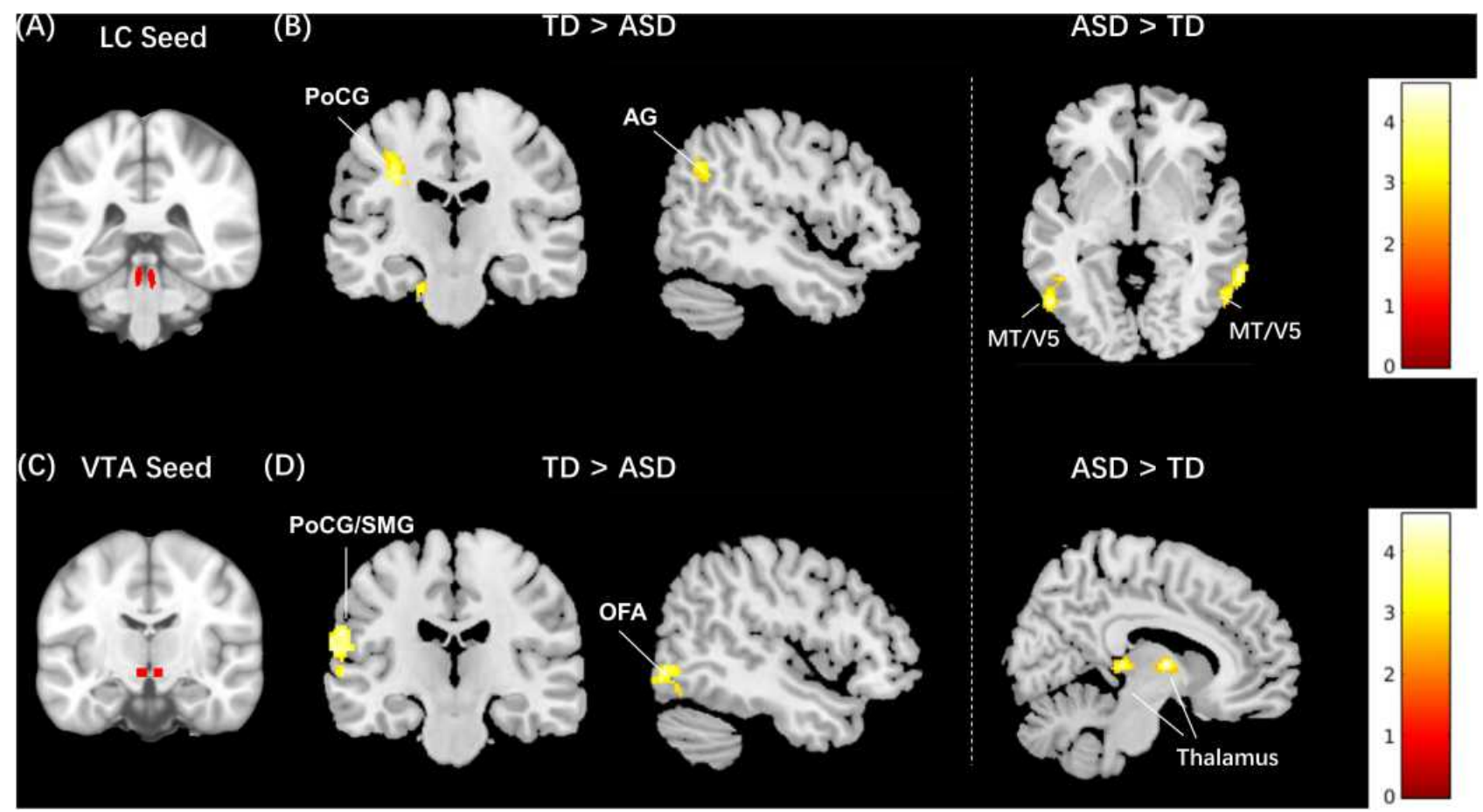

Figure I Between-group resting-state functional connectivity results of the LC and VTA as seed. (A) LC seed region; (B) middle and right panel: brain regions that showed decreased and increased functional connectivity to the LC in ASD boys compared to typically developing (TD); the color bar indicates the range of $t$ value; (C) VTA seed region; (D) middle and right panel: brain regions that showed decreased and increased functional connectivity to the VTA in ASD boys compared to typically developing (TD); the color bar indicates the range of $t$ value. 


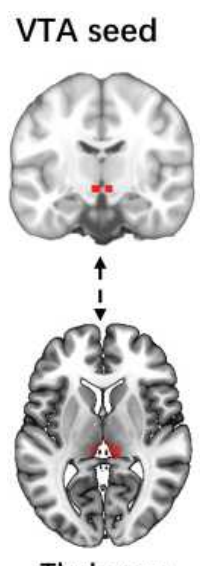

Thalamus
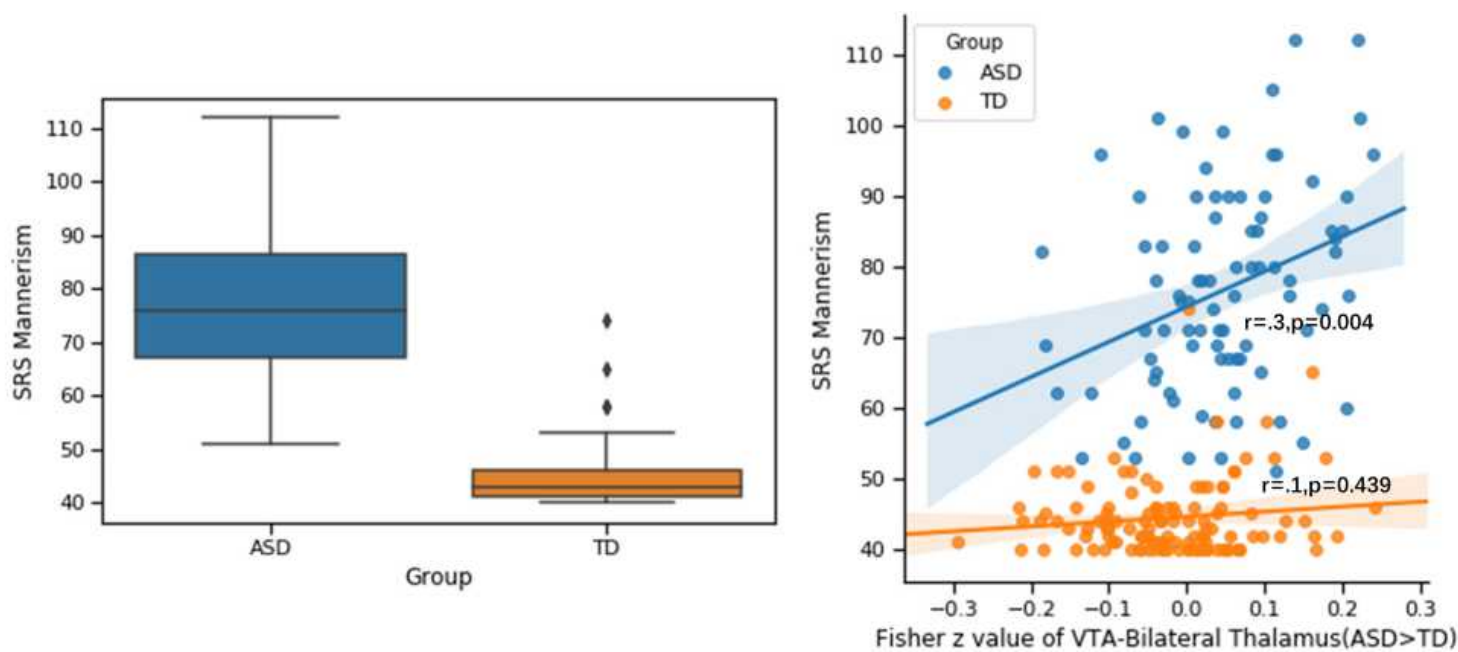

Figure 2 Left panel: demonstrating increased connectivity between VTA and bilateral thalamus in boys with ASD; middle panel: boxplot of SRS restrictive and repetitive behaviors subscore between ASD and TD group; right panel: scatterplot of VTA-thalamus connectivity with significant correlation to SRS restricted and repetitive behavior subscore (ASD: $r=0.3, p=0.004, N=86$; TD: $r=0.1, p=0.439, N=\mid 18$, adjusted for covariates).

\section{Shared LC and VTA Functional Connectivity Alternation in Boys with ASD}

Resting-state functional MRI is increasingly applied to study the organization of core processing systems of the brain. The rationale behind these studies is that the brain is always active and shows spontaneous neuronal activity even during rest. ${ }^{44,45}$ Brain regions that show synchronous neural activity are considered to be functionally connected, thereby constituting a functional network. $^{46}$

We found reduced functional connectivity of both the LC and VTA in the PoCG in boys with ASD. The PoCG is the location of the primary somatosensory cortex, the brain region that primarily processes tactile and other sensations. ${ }^{47}$ Tactile information provides feedback from the surrounding world and allows for the refinement of motor skills, visual coordination, postural responses, social behaviors and communications through early childhood. $^{48-51}$ Atypical sensory processing in ASD may contribute to social, communication, and repetitive behavior deficits, ${ }^{52}$ which are core features of autism. A structural brain imaging study found that the agerelated change in cortical thickness of postcentral gyrus was different in ASD individuals compared to TD. ${ }^{53}$ In the current study, the altered connectivity between LC-PoCG and VTA-PoCG may reflect the potential roles of NE and DA in modulating the function of the somatosensory cortex in autism individuals.

\section{Different LC and VTA Functional Connectivity Alterations in Boys with ASD}

Our results also showed an increased connectivity between the LC and MT/V5 in boys with ASD compared to the TD group. A primate study revealed that the MT/V5 may play a major role in biological motion perception, integrating local motion signals to global concepts and guiding eye movements to biological agents. ${ }^{54-56}$ Atypical biological motion perceptual processing has been widely documented in individuals with ASD. ${ }^{57}$ Nevertheless, the performance of motion processing in autism has been controversial. Some studies have suggested widespread impairments reflected in both coherent and biological motion perception in individuals with ASD. ${ }^{58,59}$ However, other studies have suggested that individuals with ASD demonstrated hypersensitivity to motion perception and enhanced integration of motion information. ${ }^{60,61}$ These results indicated that the LC may also play a role in atypical biological motion perception and processing patterns in autistic individuals. Future task-based functional imaging studies are needed to validate the role of the LC in atypical motion perception in autism.

Our results also showed that boys with ASD had decreased LC connectivity with the right AG. The AG functions as an important connector hub for multiple systems. ${ }^{53}$ Accumulating evidence from functional imaging studies indicates that the AG plays a critical role in 
various aspects of social networks such as the theory-ofmind, default mode network, and attention networks. ${ }^{54}$ The right $A G$ is especially involved in attention shifts, functioning as a "circuit breaker" for engaged attention and enabling an efficient joint attention in social interactions. ${ }^{55,56}$

Analysis of the VTA seed revealed increased connectivity with the bilateral thalamus in boys with ASD compared to the TD group. Seated in the dorsal part of the diencephalon, the thalamus functions as a pivotal hub for the relay and integration of sensory information. ${ }^{62,63}$ It also has significant roles in motor activity, emotion, memory, arousal, and other sensorimotor association function. ${ }^{64-66}$ In a previous study investigating thalamus connectivity in ASD during mildly aversive sensory input (white noise and scratchy texture), Green and colleagues ${ }^{67}$ found that individuals with ASD displayed aberrant modulation of connectivity between the pulvinar nucleus of the thalamus and sensory-motor regions compared to their TD peers. Deficits in modulation of thalamocortical connectivity in youth with ASD may reflect reduced thalamocortical inhibitions in response to sensory stimulation, which could lead to difficulty filtering out and/or integrating sensory information. As DA innervation is especially prominent in brain areas such as limbic and motor thalamic nuclei, ${ }^{68}$ increased DA innervation in the thalamus may be a possible explanation for the atypical motor behaviors observed in individuals with ASD, such as restricted and repetitive behaviors. This interpretation is further supported by the connectivitybehavior results showing that the increased VTAthalamus connections were highly associated with the impaired restrictive and repetitive behaviors in ASD group.

We also found decreased VTA connectivity with the right OFA. The OFA has long been reported to play an important role in face perception and recognition with a right hemisphere advantage, and has consistently shown to have altered activation in both resting and taskbased fMRI studies on individuals with ASD. ${ }^{69,70}$ This result is consistent with a previous imaging study, which suggested that the mesolimbic reward pathway linking the VTA and nucleus accumbens during face processing relative to scenes processing was disrupted in children with ASD. ${ }^{13}$

One limitation of this study is that the heterogeneity of ASD is not fully represented. For instance, this cohort consisted of male participants only. Thus, it is not representative of the entire ASD population (male: female $=$ $3: 1){ }^{71}$ Future studies with larger samples examining the heterogeneity might be helpful in guiding treatment approaches targeting NE and DA. Furthermore, we did not incorporate participants' medication usage ${ }^{72}$ into data analysis due to incomplete reports. Although stimulant medications were discontinued 24 hours prior to the MRI scan, future studies controlling for potential medication effects on functional connectivity need to be conducted. Another limitation of the study is the inevitable variability of scan procedure and parameters in different institutions. We have included the data sites and head motion measurements as covariates to adjust for these potential confounding factors in data analysis. Finally, task-based approaches such as incorporating medicine administration (eg, propranolol) ${ }^{32,73}$ or data-driven methods (such as independent component analysis, ICA) are desired to further understand the role of $\mathrm{NE}$ and DA systems in autism beyond the limited information from the seed-based resting-state functional connectivity study.

In conclusion, our results endorse the important role of the LC and VTA in the pathophysiology of ASD, which may shed light on future pharmacological studies targeting NE and DA for the treatment of autism.

\section{Acknowledgments}

Yiting Huang and Siyi Yu are supported by Chinese Scholarship Council (YH: 201706557085, SY: 201708510138). We wish to thank all sites and investigators who have shared their data through ABIDE. All data accessed in the ABIDE complies with relevant data protection and privacy regulations.

\section{Disclosure}

J.K has a disclosure to report: holding equity in a startup company (MNT) and pending patents to develop new neuromodulation tools; but declares no conflicts of interest for this work. All other authors declare no conflicts of interest for this work.

\section{References}

1. Fakhoury M. Autistic spectrum disorders: a review of clinical features, theories and diagnosis. Int $J$ Dev Neurosci. 2015;43:70-77. doi:10.1016/j.ijdevneu.2015.04.003

2. Muhle RA, Reed HE, Stratigos KA, Veenstra-VanderWeele J. The emerging clinical neuroscience of autism spectrum disorder: a review. JAMA Psychiatry. 2018;75(5):514-523. doi:10.1001/jamapsychiatry.2017.4685 
3. Berridge CW, Waterhouse BD. The locus coeruleus-noradrenergic system: modulation of behavioral state and state-dependent cognitive processes. Brain Res Rev. 2003;42(1):33-84.

4. Counts SE, Mufson EJ. Chapter 12 - locus coeruleus. In: Mai JK, Paxinos G, editors. The Human Nervous System. 3rd ed. San Diego: Academic Press; 2012:425-438.

5. Aston-Jones G, Rajkowski J, Cohen J. Role of locus coeruleus in attention and behavioral flexibility. Biol Psychiatry. 1999;46 (9):1309-1320. doi:10.1016/S0006-3223(99)00140-7

6. Aston-Jones G, Rajkowski J, Cohen J. Locus coeruleus and regulation of behavioral flexibility and attention. Prog Brain Res. 2000;126:165-182.

7. Bast N, Poustka L, Freitag CM. The locus coeruleus-norepinephrine system as pacemaker of attention - a developmental mechanism of derailed attentional function in autism spectrum disorder. Eur J Neurosci. 2018;47(2):115-125. doi:10.1111/ejn.13795

8. Arias-Carrion O, Poppel E. Dopamine, learning, and reward-seeking behavior. Acta Neurobiol Exp. 2007;67(4):481-488.

9. Bjorklund A, Dunnett SB. Dopamine neuron systems in the brain: an update. Trends Neurosci. 2007;30(5):194-202. doi:10.1016/j. tins.2007.03.006

10. Arias-Carrion O, Stamelou M, Murillo-Rodriguez E, MenendezGonzalez M, Poppel E. Dopaminergic reward system: a short integrative review. Int Arch Med. 2010;3:24. doi:10.1186/1755-7682-3-24

11. Halliday G, Reyes S, Double K. Chapter 13 - substantia Nigra, ventral tegmental area, and retrorubral fields. In: Mai JK, Paxinos G, editors. The Human Nervous System. 3rd ed. San Diego: Academic Press; 2012:439-455.

12. Abrams DA, Lynch CJ, Cheng KM, et al. Underconnectivity between voice-selective cortex and reward circuitry in children with autism. Proc Natl Acad Sci U S A. 2013;110(29):12060-12065. doi:10.1073/ pnas. 1302982110

13. Supekar K, Kochalka J, Schaer M, et al. Deficits in mesolimbic reward pathway underlie social interaction impairments in children with autism. Brain. 2018;141(9):2795-2805. doi:10.1093/brain/ awy191

14. Shelkar GP, Kumar S, Singru PS, Subhedar NK, Kokare DM. Noradrenergic inputs from locus coeruleus to posterior ventral tegmental area are essential to support ethanol reinforcement. Addict Biol. 2017;22(2):291-302. doi:10.1111/adb.12321

15. Guiard BP, El Mansari M, Blier P. Cross-talk between dopaminergic and noradrenergic systems in the rat ventral tegmental area, locus ceruleus, and dorsal hippocampus. Mol Pharmacol. 2008;74 (5):1463-1475. doi:10.1124/mol.108.048033

16. Park J, Wheeler RA, Fontillas K, Keithley RB, Carelli RM, Wightman RM. Catecholamines in the bed nucleus of the stria terminalis reciprocally respond to reward and aversion. Biol Psychiatry. 2012;71(4):327-334. doi:10.1016/j.biopsych.2011.10.017

17. Ventura R, Alcaro A, Puglisi-Allegra S. Prefrontal cortical norepinephrine release is critical for morphine-induced reward, reinstatement and dopamine release in the nucleus accumbens. Cerebral Cortex. 2005;15(12):1877-1886.

18. Ventura R, Cabib S, Alcaro A, Orsini C, Puglisi-Allegra S. Norepinephrine in the prefrontal cortex is critical for amphetamine-induced reward and mesoaccumbens dopamine release. J Neurosci. 2003;23(5):1879-1885. doi:10.1523/JNEURO SCI.23-05-01879.2003

19. Ventura R, Morrone C, Puglisi-Allegra S. Prefrontal/accumbal catecholamine system determines motivational salience attribution to both reward- and aversion-related stimuli. Proc Natl Acad Sci U S A. 2007;104(12):5181-5186. doi:10.1073/ pnas.0610178104

20. Xing B, Li YC, Gao WJ. Norepinephrine versus dopamine and their interaction in modulating synaptic function in the prefrontal cortex. Brain Res. 2016;1641(Pt B):217-233.
21. Dang LC, O'Neil JP, Jagust WJ. Dopamine supports coupling of attention-related networks. $J$ Neurosci. 2012;32(28):9582-9587. doi:10.1523/JNEUROSCI.0909-12.2012

22. Whitfield-Gabrieli S, Nieto-Castanon A. Conn: a functional connectivity toolbox for correlated and anticorrelated brain networks. Brain Connect. 2012;2(3):125-141. doi:10.1089/brain.2012.0073

23. Mittner M, Hawkins GE, Boekel W, Forstmann BU. A neural model of mind wandering. Trends Cogn Sci. 2016;20(8):570-578. doi:10.1016/j.tics.2016.06.004

24. Lam KS, Aman MG, Arnold LE. Neurochemical correlates of autistic disorder: a review of the literature. Res Dev Disabil. 2006;27 (3):254-289. doi:10.1016/j.ridd.2005.03.003

25. Lake CR, Ziegler MG, Murphy DL. Increased norepinephrine levels and decreased dopamine-beta-hydroxylase activity in primary autism. Arch Gen Psychiatry. 1977;34(5):553-556. doi:10.1001/ archpsyc.1977.01770170063005

26. London EB. Neuromodulation and a reconceptualization of autism spectrum disorders: using the locus coeruleus functioning as an exemplar. Front Neurol. 2018;9(1120). doi:10.3389/fneur.2018.01120

27. Pavăl D. A dopamine hypothesis of autism spectrum disorder. Dev Neurosci. 2017;39(5):355-360. doi:10.1159/000478725

28. Zamzow RM, Ferguson BJ, Stichter JP, et al. Effects of propranolol on conversational reciprocity in autism spectrum disorder: a pilot, double-blind, single-dose psychopharmacological challenge study. Psychopharmacology. 2016;233(7):1171-1178. doi:10.1007/s00213015-4199-0

29. Zamzow RM, Ferguson BJ, Ragsdale AS, Lewis ML, Beversdorf DQ. Effects of acute beta-adrenergic antagonism on verbal problem solving in autism spectrum disorder and exploration of treatment response markers. J Clin Exp Neuropsychol. 2017;39 (6):596-606. doi:10.1080/13803395.2016.1252724

30. Ratey JJ, Bemporad J, Sorgi P, et al. Open trial effects of beta-blockers on speech and social behaviors in 8 autistic adults. J Autism Dev Disord. 1987;17(3):439-446. doi:10.1007/BF01487073

31. Sagar-Ouriaghli I, Lievesley K, Santosh PJ. Propranolol for treating emotional, behavioural, autonomic dysregulation in children and adolescents with autism spectrum disorders. $J$ Psychopharmacol. 2018;32(6):641-653. doi:10.1177/0269881118756245

32. Hegarty JP, Zamzow RM, Ferguson BJ, et al. Beta-adrenergic antagonism alters functional connectivity during associative processing in a preliminary study of individuals with and without autism. Autism. 2020;24(3):1362361319868633. doi:10.1177/1362361319868633

33. Narayanan A, White CA, Saklayen S, et al. Effect of propranolol on functional connectivity in autism spectrum disorder-a pilot study. Brain Imaging Behav. 2010;4(2):189-197. doi:10.1007/s11682-0109098-8

34. Baron-Cohen S, Knickmeyer RC, Belmonte MK. Sex differences in the brain: implications for explaining autism. Science. 2005;310 (5749):819-823. doi:10.1126/science.1115455

35. Dickstein DP, Pescosolido MF, Reidy BL, et al. Developmental meta-analysis of the functional neural correlates of autism spectrum disorders. J Am Acad Child Adolesc Psychiatry. 2013;52(3):279-289. doi:10.1016/j.jaac.2012.12.012

36. Constantino JN, Davis SA, Todd RD, et al. Validation of a brief quantitative measure of autistic traits: comparison of the social responsiveness scale with the autism diagnostic interview-revised. $J$ Autism Dev Disord. 2003;33(4):427-433. doi:10.1023/ A:1025014929212

37. Jenkinson M, Bannister P, Brady M, Smith S. Improved optimization for the robust and accurate linear registration and motion correction of brain images. Neuroimage. 2002;17(2):825-841. doi:10.1006/ nimg.2002.1132

38. Yan CG, Cheung B, Kelly C, et al. A comprehensive assessment of regional variation in the impact of head micromovements on functional connectomics. Neuroimage. 2013;76:183-201. doi:10.1016/j. neuroimage.2013.03.004 
39. Tona KD, Keuken MC, de Rover M, et al. In vivo visualization of the locus coeruleus in humans: quantifying the test-retest reliability. Brain Struct Funct. 2017;222(9):4203-4217. doi:10.1007/s00429017-1464-5

40. Adcock RA, Thangavel A, Whitfield-Gabrieli S, Knutson B, Gabrieli JD. Reward-motivated learning: mesolimbic activation precedes memory formation. Neuron. 2006;50(3):507-517. doi:10.1016/ j.neuron.2006.03.036

41. MacInnes JJ, Dickerson KC, Chen NK, Adcock RA. Cognitive neurostimulation: learning to volitionally sustain ventral tegmental area activation. Neuron. 2016;89(6):1331-1342. doi:10.1016/j. neuron.2016.02.002

42. Carter RM, Macinnes JJ, Huettel SA, Adcock RA. Activation in the VTA and nucleus accumbens increases in anticipation of both gains and losses. Front Behav Neurosci. 2009;3:21. doi:10.3389/ neuro.08.021.2009

43. Kahn I, Shohamy D. Intrinsic connectivity between the hippocampus, nucleus accumbens, and ventral tegmental area in humans. Hippocampus. 2013;23(3):187-192. doi:10.1002/hipo.22077

44. Barkhof F, Haller S, Rombouts SA. Resting-state functional MR imaging: a new window to the brain. Radiology. 2014;272 (1):29-49. doi:10.1148/radiol.14132388

45. van den Heuvel MP, Hulshoff Pol HE. Exploring the brain network: a review on resting-state fMRI functional connectivity. Eur Neuropsychopharmacol. 2010;20(8):519-534. doi:10.1016/j. euroneuro.2010.03.008

46. Damoiseaux JS, Rombouts SA, Barkhof F, et al. Consistent resting-state networks across healthy subjects. Proc Natl Acad Sci U S A. 2006;103(37):13848-13853. doi:10.1073/pnas.0601417103

47. Dharani K. Chapter 1 - functional anatomy of the brain. In: Dharani K, editor. The Biology of Thought. San Diego: Academic Press; 2015:3-29.

48. Cascio CJ, Moore D, McGlone F. Social touch and human development. Dev Cogn Neurosci. 2019;35:5-11. doi:10.1016/j. den.2018.04.009

49. Damasio AR. The somatic marker hypothesis and the possible functions of the prefrontal cortex. Philos Trans $R$ Soc Lond B Biol Sci. 1996;351(1346):1413-1420.

50. Keysers C, Kaas JH, Gazzola V. Somatosensation in social perception. Nat Rev Neurosci. 2010;11(6):417-428. doi:10.1038/ nrn2833

51. Kragel PA, LaBar KS. Somatosensory representations link the perception of emotional expressions and sensory experience. eNeuro. 2016;3(2):ENEURO.0090-0015.2016. doi:10.1523/ENEURO.009015.2016

52. Foss-Feig JH, Heacock JL, Cascio CJ. Tactile responsiveness patterns and their association with core features in autism spectrum disorders. Res Autism Spectr Disord. 2012;6(1):337-344. doi:10.1016/j. rasd.2011.06.007

53. Zielinski BA, Prigge MB, Nielsen JA, et al. Longitudinal changes in cortical thickness in autism and typical development. Brain. 2014;137(Pt 6):1799-1812. doi:10.1093/brain/awu083

54. Born RT, Bradley DC. Structure and function of visual area MT. Annu Rev Neurosci. 2005;28:157-189. doi:10.1146/annurev. neuro.26.041002.131052

55. Zeki S. Area V5-a microcosm of the visual brain. Front Integr Neurosci. 2015;9:21. doi:10.3389/fnint.2015.00021

56. Watson JD, Myers R, Frackowiak RS, et al. Area V5 of the human brain: evidence from a combined study using positron emission tomography and magnetic resonance imaging. Cerebral Cortex. 1993;3(2):79-94.
57. Ben-Sasson A, Hen L, Fluss R, Cermak SA, Engel-Yeger B, Gal E. A meta-analysis of sensory modulation symptoms in individuals with autism spectrum disorders. J Autism Dev Disord. 2009;39(1):1-11. doi:10.1007/s10803-008-0593-3

58. Spencer J, O’Brien J, Riggs K, Braddick O, Atkinson J, Wattam-Bell J. Motion processing in autism: evidence for a dorsal stream deficiency. Neuroreport. 2000;11(12):2765-2767. doi:10.1097/ 00001756-200008210-00031

59. Koldewyn K, Whitney D, Rivera SM. Neural correlates of coherent and biological motion perception in autism. Dev Sci. 2011;14 (5):1075-1088. doi:10.1111/j.1467-7687.2011.01058.x

60. Foss-Feig JH, Tadin D, Schauder KB, Cascio CJ. A substantial and unexpected enhancement of motion perception in autism. $J$ Neurosci. 2013;33(19):8243-8249. doi:10.1523/JNEUROSCI.1608-12.2013

61. Manning C, Tibber MS, Charman T, Dakin SC, Pellicano E. Enhanced integration of motion information in children with autism. $J$ Neurosci. 2015;35(18):6979-6986. doi:10.1523/ JNEUROSCI.4645-14.2015

62. Guillery RW, Sherman SM. Thalamic relay functions and their role in corticocortical communication: generalizations from the visual system. Neuron. 2002;33(2):163-175. doi:10.1016/S0896-6273(01) 00582-7

63. Mitchell AS, Sherman SM, Sommer MA, Mair RG, Vertes RP, Chudasama Y. Advances in understanding mechanisms of thalamic relays in cognition and behavior. $J$ Neurosci. 2014;34 (46):15340-15346. doi:10.1523/JNEUROSCI.3289-14.2014

64. Pessoa L. A network model of the emotional brain. Trends Cogn Sci. 2017;21(5):357-371. doi:10.1016/j.tics.2017.03.002

65. Sommer MA. The role of the thalamus in motor control. Curr Opin Neurobiol. 2003;13(6):663-670. doi:10.1016/j.conb.2003.10.014

66. Halassa MM, Kastner S. Thalamic functions in distributed cognitive control. Nat Neurosci. 2017;20(12):1669-1679. doi:10.1038/s41593017-0020-1

67. Green SA, Hernandez L, Bookheimer SY, Dapretto M. Reduced modulation of thalamocortical connectivity during exposure to sensory stimuli in ASD. Autism Res. 2017;10(5):801-809. doi:10.1002/ aur. 1726

68. Sanchez-Gonzalez MA, Garcia-Cabezas MA, Rico B, Cavada C. The primate thalamus is a key target for brain dopamine. $J$ Neurosci. 2005;25(26):6076-6083. doi:10.1523/JNEUROSCI.0968-05.2005

69. Kleinhans NM, Richards T, Sterling L, et al. Abnormal functional connectivity in autism spectrum disorders during face processing. Brain. 2008;131(Pt 4):1000-1012. doi:10.1093/brain/awm334

70. Pierce K, Muller RA, Ambrose J, Allen G, Courchesne E. Face processing occurs outside the fusiform 'face area' in autism: evidence from functional MRI. Brain. 2001;124(Pt 10):2059-2073. doi:10.1093/brain/124.10.2059

71. Loomes R, Hull L, Mandy WPL. What is the male-to-female ratio in autism spectrum disorder? A systematic review and meta-analysis. J Am Acad Child Adolesc Psychiatry. 2017;56(6):466-474. doi:10.1016/j.jaac.2017.03.013

72. Linke AC, Olson L, Gao Y, Fishman I, Müller R-A. Psychotropic medication use in autism spectrum disorders may affect functional brain connectivity. Biol Psychiatry Cogn Neurosci Neuroimaging. 2017;2(6):518-527. doi:10.1016/j.bpsc.2017.06.008

73. Rasmussen J, Casey BJ, van Erp TG, et al. ADHD and cannabis use in young adults examined using fMRI of a Go/NoGo task. Brain Imaging Behav. 2016;10(3):761-771. doi:10.1007/s11682-015-9438-9 


\section{Publish your work in this journal}

Neuropsychiatric Disease and Treatment is an international, peerreviewed journal of clinical therapeutics and pharmacology focusing on concise rapid reporting of clinical or pre-clinical studies on a range of neuropsychiatric and neurological disorders. This journal is indexed on PubMed Central, the 'PsycINFO' database and CAS, and is the official journal of The International Neuropsychiatric Association (INA). The manuscript management system is completely online and includes a very quick and fair peer-review system, which is all easy to use. Visit http://www.dovepress.com/testimonials.php to read real quotes from published authors.

Submit your manuscript here: https://www.dovepress.com/neuropsychiatric-disease-and-treatment-journal 